\title{
A Central Limit Theorem for the Subadditive Process and Its Application to Products of Random Matrices
}

\author{
By \\ Hiroshi IsHitanI*
}

\section{$\S 0$. Introduction}

The purpose of the present paper is to give a central limit theorem for subadditive process in the sense of J. F. C. Kingman (cf. [3], [4]).

Throughout this article $(\Omega, \mathscr{B}, P)$ denotes a probability space on which all random variables are defined. Let $T$ be a measure preserving transformation in what follows. According to Kingman, a family $\left(x_{s, t}\right.$; $s<t, s=0,1, \ldots, t=1,2, \ldots)$ of random variables is called a subadditive process, if the following three conditions $\mathbf{S}_{1}, \mathbf{S}_{2}$ and $\mathbf{S}_{3}$ are satisfied.

$\mathbf{S}_{1} . \quad x_{s, u} \leqq x_{s, t}+x_{t, u}$ for all $s<t<u$.

$\mathbf{S}_{2} . \quad x_{s+1, t+1}(\omega)=x_{s, i}(T \omega)$ for all $s<t$.

$\mathbf{S}_{3}$. The expectation $E\left(x_{0, t}\right)$ exists and satisfies

$$
E\left(x_{0, t}\right) \geqq-A t,
$$

for all $t \geqq 1$ with some constant $A$.

In $\S 1$ and $\S 2$ we show that the random variable

$$
t^{-\frac{1}{2}\left(x_{0, t}-E\left(x_{0, t}\right)\right)}
$$

has an asymptotically normal distribution under suitable conditions. J. F. C. Kingman and D. L. Burkholder proved the decomposition of

Communicated by K. Itô, December 24, 1974. Revised June 29, 1976.

* Department of Mathematics, Kobe University, Kobe.

Present address, Department of Mathematics, Mie University, Tsu, Mie. 
subadditive processes into additive ones (cf. [3], [4]). Hence we can reduce our problem to the central limit theorem for stationary sequences given in [2].

In $\$ 3$ we treat an application to products of positive random matrices. H. Furstenberg and H. Kesten have already obtained a central limit theorem for those (cf. [1]). By using the result in $\S 1$, we can weaken their conditions on moments and "weak dependence".

The author would like to express his hearty thanks to Prof. M. Nisio and Prof. T. Shiga for their encouragement and discussions.

\section{§1. Notations and Results}

In order to state our results, we shall introduce some notations. Set $g_{t}=E\left(x_{0, t}\right)$ and $\gamma=\lim _{t \rightarrow \infty} g_{t} / t$ (cf. [4], p. 883). Let $\left\{\mathscr{M}_{a}^{b} ; a \leqq b, a=0\right.$, $1, \ldots, \infty, b=0,1, \ldots, \infty\}$ be $e^{t \rightarrow \infty}$ family of sub- $\sigma$-fields of $\mathscr{B}$ satisfying the following two conditions $\mathbf{P}_{1}$ and $\mathbf{P}_{2}$ :

$\mathbf{P}_{1}$. If $a \leqq c \leqq d \leqq b$, then $\mathscr{M}_{c}^{d} \subset \mathscr{M}_{a}^{b}$.

$\mathbf{P}_{2}$. For all $a \leqq b, T^{-1} \mathscr{M}_{a}^{b}=\mathscr{M}_{a+1}^{b+1}$.

We define $\phi(n)$ and $\alpha(n)$ by

(1.1) $\phi(n)=\sup _{k \geqq 0} \sup \left\{\frac{|P(A \cap B)-P(A) P(B)|}{P(A)} ; A \in \mathscr{M}_{0}^{k}, B \in \mathscr{M}_{k+n}^{\infty}, P(A) \neq 0\right\}$, and

$(1 \cdot 2) \quad \alpha(n)=\sup _{k \geqq 0} \sup \left\{|P(A \cap B)-P(A) P(B)| ; A \in \mathscr{M}_{0}^{k}, B \in \mathscr{M}_{k+n}^{\infty}\right\}$.

Let us define $\Phi_{\sigma}(z)$ by

$$
\Phi_{\sigma}(z)=\frac{1}{\sqrt{2 \pi} \sigma} \int_{-\infty}^{z} \exp \left[-t^{2} / 2 \sigma^{2}\right] d t
$$

for $\sigma>0$ and

$$
\Phi_{0}(z)= \begin{cases}1, & (z>0) \\ 0, & (z \leqq 0)\end{cases}
$$


The notation $\|*\|_{\theta}(\theta \geqq 1)$ stands for $\left[E|*|^{\theta}\right]^{1 / \theta}$.

We are now in a position to state our results.

Theorem 1. Let $\left(x_{s, t}\right)$ be a subadditive process and $\left\{\mathscr{M}_{a}^{b}\right\}$ be a family of sub- $\sigma$-fields of $\mathscr{B}$ satisfying $\mathbf{P}_{1}$ and $\mathbf{P}_{2}$. Suppose that the following four conditions are satisfied:

(1) $\left(g_{t}-t \gamma\right) / \sqrt{t} \rightarrow 0$, as $t \rightarrow \infty$,

(2) $\sum_{n=1}^{\infty}[\phi(n)]^{\frac{1}{2}}<+\infty$,

(3) there exists a random variable $\Psi$ such that $E|\Psi|^{2}<+\infty$ and

$$
\left|x_{0, t}-x_{1, t}\right| \leqq \Psi \quad \text { (a.e.) }
$$

for all $t \geqq 1$,

(4) $\sum_{n=1}^{\infty} \sup _{t \geqq 1}\left\|\left(x_{0, t}-x_{1, t}\right)-E\left(x_{0, t}-x_{1, t} \mid \mathscr{M}_{0}^{n}\right)\right\|_{2}<+\infty$.

Then we have, for some $\sigma \geqq 0$,

$$
\lim _{t \rightarrow \infty} P\left\{\frac{1}{\sqrt{t}}\left(x_{0, t}-g_{t}\right)<z\right\}=\Phi_{\sigma}(z)
$$

at every continuity point of $\Phi_{\sigma}(z)$.

If we assume a stronger condition than (3) of Theorem 1, we can weaken the conditions (2) and (4); namely we have the following remarks.

Remark 1. The conclusion of Theorem 1 remains valid if the conditions (2)-(4) of Theorem 1 are replaced by

(2') $\sum_{n=1}^{\infty}[\alpha(n)]^{\delta /(2+\delta)}<+\infty$ for some $\delta>0$,

(3') there exists a random variable $\Psi$ such that $E|\Psi|^{2+\delta}<+\infty$ and

$$
\left|x_{0, t}-x_{1, t}\right| \leqq \Psi \quad \text { (a.e.) }
$$

for all $t \geqq 1$, 
$\left(4^{\prime}\right) \sum_{n=1}^{\infty} \sup _{t \geqq 1}\left\|\left(x_{0, t}-x_{1, t}\right)-E\left(x_{0, t}-x_{1, t} \mid \mathscr{M}_{0}^{n}\right)\right\|_{\theta}<+\infty$,

where $\theta=(2+\delta) /(1+\delta)$.

Remark 2. The conclusion of Theorem 1 remains valid if the conditions (2)-(4) of Theorem 1 are replaced by

(2") $\sum_{n=1}^{\infty} \alpha(n)<+\infty$

$\left(3^{\prime \prime}\right)$ there exists an essentially bounded random variable $\Psi$ such that

$$
\left|x_{0, t}-x_{1, t}\right| \leqq \Psi \quad \text { (a.e.) }
$$

for all $t \geqq 1$,

$\left(4^{\prime \prime}\right) \quad \sum_{n=1}^{\infty} \sup _{t \geqq 1}\left\|\left(x_{0, t}-x_{1, t}\right)-E\left(x_{0, t}-x_{1, t} \mid \mathscr{l}_{0}^{n}\right)\right\|_{1}<+\infty$.

\section{§2. Proof of Theorem 1}

Our result can be easily obtained by combining the following two theorems. The first theorem is proved by J. F. C. Kingman and D. L. Burkholder (cf. [3], [4]). The second is a central limit theorem for stationary sequences of weakly dependent case, which can be found in [2].

Theorem A. There is a sequence $n_{1}<n_{2}<\cdots$ of positive integers and an integrable random variable $y$ such that

$$
y=\lim _{N \rightarrow \infty} \frac{1}{N} \sum_{j=1}^{N} \frac{1}{n_{j}} \sum_{t=1}^{n_{j}}\left(x_{0, t}-x_{1, t}\right) \quad \text { (a.e.). }
$$

Moreover we have $E y=\gamma$ and

$$
\sum_{k=s}^{t-1} y\left(T^{k} \omega\right) \leqq x_{s, t}(\omega)
$$

Theorem $\mathbb{B}$ (Theorem $18 \cdot 6 \cdot 1$ in [2]). Let $y$ be a random variable and $T$ be a measure preserving transformation. Let $\left\{\mathscr{M}_{a}^{b}\right\}$ be a family of sub- $\sigma$-fields satisfying $\mathbb{P}_{1}$ and $\mathbb{P}_{2}$. Suppose that 
(1) $\sum_{n=1}^{\infty}[\phi(n)]^{\frac{1}{2}}<+\infty$

(2) $E|y|^{2}<+\infty$,

(3) $\sum_{n=1}^{\infty}\left\|y-E\left(y \mid \mathscr{M}_{0}^{n}\right)\right\|_{2}<+\infty$.

Then there exists a nonnegative constant $\sigma$ and we have

$$
\lim _{n \rightarrow \infty} P\left\{\frac{1}{\sqrt{n}}\left(\sum_{h=0}^{n-1} y\left(T^{k} \omega\right)-n E(y)\right)<z\right\}=\Phi_{\sigma}(z)
$$

at every continuity point of $\Phi_{\sigma}(z)$.

Proof of Theorem 1. By virtue of Theorem A, we can get

$$
\begin{aligned}
& E \mid \frac{1}{\sqrt{t}}\left(x_{0, t}(\omega)-g_{t}\right)-\frac{1}{\sqrt{t}}\left(\sum_{h=0}^{t-1} y\left(T^{h}(\omega)-t \gamma\right) \mid\right. \\
& \quad \leqq \frac{1}{\sqrt{t}}\left(g_{t}-t \gamma\right)+E\left|\frac{1}{\sqrt{t}}\left(x_{0, t}(\omega)-\sum_{k=0}^{t-1} y\left(T^{k} \omega\right)\right)\right|=-\frac{2}{\sqrt{t}}\left(g_{t}-t \gamma\right)
\end{aligned}
$$

On the other hand, from the condition (1) of Theorem 1, we have

$$
\frac{2}{\sqrt{t}}\left(g_{t}-t \gamma\right) \longrightarrow 0
$$

as $1 \rightarrow \infty$. Hence our theorem can be deduced from the central limit theorem for the stationary sequence $\left\{y\left(T^{k} \omega\right) ; k=0,1, \ldots\right\}$.

The condition (2) of Theoren 1 immediately implies (1) of Theorem $B$, so we only need to check that $y$ satisfies the conditions (2) and (3) of Theorem B. Using the condition (3) of Theorem 1 and $(2 \cdot 1)$, we have

$$
E|y|^{2} \leqq E\left(\lim _{N \rightarrow \infty} \frac{1}{N} \sum_{j=1}^{N} \frac{1}{n_{j}} \sum_{t=1}^{n_{j}}\left|x_{0, t}-x_{1, t}\right|\right)^{2} \leqq E|\Psi|^{2}<+\infty,
$$

which implies the condition (2) of Theorem B.

Moreover the condition (3) of Theorem 1 guarantees that

$$
E\left(y \mid \mathscr{U}_{0}^{n}\right)=\lim _{N \rightarrow \infty} \frac{1}{N} \sum_{j=1}^{N} \frac{1}{n_{j}} \sum_{t=1}^{n_{j}} E\left(x_{0, t}-x_{1, t} \mid \mathscr{C}_{0}^{n}\right)
$$


and

$$
\begin{aligned}
& \left|\frac{1}{N} \sum_{j=1}^{N} \frac{1}{n_{j}} \sum_{t=1}^{n_{j}}\left\{\left(x_{0, t}-x_{1, t}\right)-E\left(x_{0, t}-x_{1, t} \mid \mathscr{M}_{0}^{n}\right)\right\}\right| \\
& \quad \leqq \Psi+E\left(\Psi \mid \mathscr{M}_{0}^{n}\right) .
\end{aligned}
$$

Therefore we obtain, from $(2 \cdot 3)$ and $(2 \cdot 4)$,

$$
\begin{aligned}
\| y & -E\left(y \mid \mathscr{M}_{0}^{n}\right) \|_{2} \\
& =\lim _{N \rightarrow \infty}\left\|\frac{1}{N} \sum_{j=1}^{N} \frac{1}{n_{j}} \sum_{t=1}^{n_{j}}\left\{\left(x_{0, t}-x_{1, t}\right)-E\left(x_{0, t}-x_{1, t} \mid \mathscr{M}_{0}^{n}\right)\right\}\right\|_{2} \\
(2 \cdot 5) \quad & \leqq \lim _{N \rightarrow \infty} \frac{1}{N} \sum_{j=1}^{N} \frac{1}{n_{j}} \sum_{t=1}^{n_{j}}\left\|\left(x_{0, t}-x_{1, t}\right)-E\left(x_{0, t}-x_{1, t} \mid \mathscr{M}_{0}^{n}\right)\right\|_{2} \\
& \leqq \sup _{t \geqq 1}\left\|\left(x_{0, t}-x_{1, t}\right)-E\left(x_{0, t}-x_{1, t} \mid \mathscr{M}_{0}^{n}\right)\right\|_{2} .
\end{aligned}
$$

Combining (2.5) and the condition (4) of Theorem 1, we get that the condition (3) of Theorem B holds for $y$. The proof is therefore complete.

Remark 3. Remarks 1 and 2 in $\S 1$ can be deduced from Theorems $18 \cdot 6 \cdot 2$ and $18 \cdot 6 \cdot 3$ in [2], instead of Theorem B, respectively. The proof is exactly similar to the proof of Theorem 1 .

\section{§3. Application to Products of Random Matrices}

Let $Z^{1}, Z^{2}, \ldots$ be a stationary sequence of random $k \times k$ matrices with strictly positive elements. By the stationarity there is a measure preserving transformation $T$ such that

$$
Z^{i+1}(\omega)=Z^{i}(T \omega) \quad(i=1,2, \ldots)
$$

Let us define a family of sub- $\sigma$-fields as follows:

$$
\mathscr{M}_{a}^{b}=\sigma\left\{Z^{a+1}, Z^{a+2}, \ldots, Z^{b+1}\right\}
$$

where the notation $\sigma\{*\}$ stands for the $\sigma$-field generated by $*$. Clearly, this $\left\{\mathscr{M}_{a}^{b}\right\}$ satisfies $\mathbb{P}_{1}$ and $\mathbb{P}_{2}$ with respect to $T$. Associated with this 
family, let $\phi(n)$ and $\alpha(n)$ be the quantities defined by $(1 \cdot 1)$ and $(1 \cdot 2)$ respectively. We denote by $(A)_{i, j}$ the $(i, j)$-th element of a matrix $A$. We write

$$
{ }^{t} Y^{s}=Z^{t} \cdot Z^{t-1} \cdots Z^{s}
$$

Under these notations, we can obtain the following

Theorem 2. Suppose that $\left(Z^{i} ; i=1,2, \ldots\right)$ is a stationary sequence of random matrices and the elements of these matrices are strictly positive. Suppose also that

(1) there exists a positive constant $C$ such that

$$
\left.1 \leqq \max _{i, j}\left(Z^{1}\right)_{i, j} / \min _{i, j}\left(Z^{1}\right)_{i, j} \leqq C \quad \text { (a.e. }\right),
$$

(2) $\sum_{n=1}^{\infty}[\phi(n)]^{\frac{1}{2}}<+\infty$,

and

(3) $E\left|\log \left(Z^{1}\right)_{1,1}\right|^{2}<+\infty$.

Then there is a nonnegative constant $\sigma$ such that, for all $1 \leqq i, j \leqq k$,

$$
\lim _{t \rightarrow \infty} P\left\{\frac{1}{\sqrt{t}}\left[\log \left({ }^{t} Y^{1}\right)_{i, j}-E\left(\log \left({ }^{t} Y^{1}\right)_{i, j}\right)\right]<z\right\}=\Phi_{\sigma}(z)
$$

at every continuity point of $\Phi_{\sigma}(z)$.

Proof. Combining (1) and (3) of Theorem 2, we have

$$
E\left(\left|\log \left(Z^{1}\right)_{i, j}\right|\right)<+\infty
$$

for all $1 \leqq i, j \leqq k$. This fact and the positivity of elements show that the family of random variables

$$
x_{s, t}=-\log \left({ }^{t} Y^{s+1}\right)_{1,1}
$$

is a subadditive process (cf. [4], pp. 891-892).

First, we prove the asymptotic normality of the random variables

$$
\frac{1}{\sqrt{t}}\left\{x_{0, t}-E\left(x_{0, t}\right)\right\}
$$


using Theorem 1.

(a) Under the condition (1) of Theorem 2, it is already shown in [1] that

$$
g_{t+1}-g_{t}=\gamma+O\left(\left(1-C^{-3}\right)^{t}\right)
$$

([1], p. 467), where $g_{t}=E\left(x_{0, t}\right)$ and $\gamma=\lim _{t \rightarrow \infty} g_{t} / t$ as in $\S 1$. This fact allows us to deduce the condition (1) of Theorem 1.

(b) The condition (2) of Theorem 2 immediately implies (2) of Theorem 1.

(c) The obvious equality

$$
\frac{\left({ }^{t} Y^{1}\right)_{1,1}}{\left({ }^{t} Y^{2}\right)_{1,1}}=\frac{\sum_{i=1}^{k} \sum_{j=1}^{k}\left({ }^{t} Y^{3}\right)_{1, i}\left(Z^{2}\right)_{i, j}\left(Z^{1}\right)_{j, 1}}{\sum_{i=1}^{k}\left({ }^{t} Y^{3}\right)_{1, i}\left(Z^{2}\right)_{i, 1}}
$$

and the condition (1) of Theorem 2 show that the following evaluations

$$
C^{-1} \sum_{j=1}^{h}\left(Z^{1}\right)_{j, 1} \leqq \frac{\left({ }^{t} Y^{1}\right)_{1,1}}{\left({ }^{t} Y^{2}\right)_{1,1}} \leqq C \sum_{j=1}^{k}\left(Z^{1}\right)_{j, 1}
$$

Hence we have

$$
\left|x_{0, t}-x_{1, t}\right| \leqq\left|\log \left(Z^{1}\right)_{1,1}\right|+\log k C^{2}
$$

This inequality and the condition (3) of Theorem 2 allow us to derive (3) of Theorem 1.

(d) If $A, B$ and $C$ are $k \times k$ matrices and if the notation $(A)_{*, j}$ stands for $\sum_{i=1}^{k}(A)_{i, j}$, then the equality

$$
\begin{aligned}
& \frac{(A B C)_{1,1}}{(A B)_{1,1}}=\sum_{i=1}^{k} \frac{(B)_{*, i}}{(B)_{*, 1}}(C)_{i, 1} \\
& +\frac{\sum_{i=1}^{k} \sum_{j=1}^{k}(A)_{1, j}\left[\frac{(B)_{j, i}}{(B)_{*, i}}-\frac{(B)_{j, 1}}{(B)_{*, 1}}\right](B)_{*, i}(C)_{i, 1}}{\sum_{j=1}^{k}(A)_{1, j}(B)_{j, 1}}
\end{aligned}
$$

can be easily shown. Now take $A={ }^{m+n} Y^{n+1}, B={ }^{n} Y^{2}$ and $C=Z^{1}$. With these substitutions we have 


$$
\begin{aligned}
& \frac{\left({ }^{m+n} Y^{1}\right)_{1,1}}{\left({ }^{m+n} Y^{2}\right)_{1,1}}=\sum_{i=1}^{k} \frac{\left({ }^{n} Y^{2}\right)_{*, i}}{\left(n Y^{2}\right)_{*, 1}}\left(Z^{1}\right)_{1,1} \\
& +\frac{\sum_{i=1}^{k} \sum_{j=1}^{k}\left({ }^{m+n} Y^{n+1}\right)_{1, j}\left[\frac{\left(n Y^{2}\right)_{j, i}}{\left({ }^{n} Y^{2}\right)_{*, i}}-\frac{\left({ }^{n} Y^{2}\right)_{j, 1}}{\left({ }^{n} Y^{2}\right)_{*, 1}}\right]\left({ }^{n} Y^{2}\right)_{*, i}\left(Z^{1}\right)_{i, 1}}{\sum_{j=1}^{k}\left({ }^{m+n} Y^{n+1}\right)_{1, j}\left({ }^{n} Y^{2}\right)_{j, 1}}
\end{aligned}
$$

We denote by $\alpha$ the first term in the right hand side of $(3 \cdot 3)$ and by $\beta$ the second term.

The obvious identity

$$
\frac{\left({ }^{m+n} Y^{m}\right)_{i_{1}, j_{1}}}{\left({ }^{m+n} Y^{m}\right)_{i_{2}, j_{2}}}=\frac{\sum_{r, s}\left(Z^{m+n}\right)_{i_{1}, r}\left({ }^{m+n-1} Y^{m+1}\right)_{r, s}\left(Z^{m}\right)_{s, j_{1}}}{\sum_{r, s}\left(Z^{m+n}\right)_{i_{2}, r}\left({ }^{m+n-1} Y^{m+1}\right)_{r, s}\left(Z^{m}\right)_{s, j_{2}}}
$$

and the condition (1) of Theorcm 2 allow us to derive

$$
C^{-2} \leqq\left(m+n Y^{m}\right)_{i_{1}, j_{1}} /\left({ }^{m+n} Y^{m}\right)_{i_{2}, j_{2}} \leqq C^{2}
$$

as was pointed out in [1] (cf. [1], Lemma 2). From (3.4) it clearly follows that

$$
C^{-2} \leqq\left({ }^{n} Y^{2}\right)_{*, i} /\left({ }^{n} Y^{2}\right)_{*, 1} \leqq C^{2}
$$

Therefore, using the condition (1) of Theorcm 2, we have

$$
k C^{-3}\left(Z^{1}\right)_{1,1} \leqq \alpha \leqq k C^{3}\left(Z^{1}\right)_{1,1} .
$$

To deal with $\beta$, we derive

$$
\left|\frac{\left({ }^{n} Y^{2}\right)_{j, i}}{\left({ }^{n} Y^{2}\right)_{*, i}}-\frac{\left({ }^{n} Y^{2}\right)_{j, 1}}{\left({ }^{n} Y^{2}\right)_{*, 1}}\right| \leqq\left(1-C^{-3}\right)^{n-2}
$$

from $(3 \cdot 4)$ and the condition (1) of Theorem 2. The proof of $(3 \cdot 6)$ is all the same as the proof of Lemma 3 in [1], p. 463, so we omit it. Inequality $(3 \cdot 4)$ guarantees that

$$
\max _{i}\left({ }^{n} Y^{2}\right)_{*, i} / \min _{j}\left({ }^{n} Y^{2}\right)_{j, 1} \leqq k C^{2}
$$

Combining $(3 \cdot 6)$ and $(3 \cdot 7)$ allows us to deduce that

$$
\beta \leqq k C^{2}\left(1-C^{-3}\right)^{n-2} \sum_{i=1}^{k}\left(Z^{1}\right)_{i, 1} \leqq k^{2} C^{3}\left(1-C^{-3}\right)^{n-2}\left(Z^{1}\right)_{1,1}
$$


Using $(3 \cdot 5)$ and $(3 \cdot 8)$, we can get

$$
\beta / \alpha \leqq k C^{6}\left(1-C^{-3}\right)^{n-2} .
$$

Hence, from the identity

$$
\log (\alpha+\beta)=\log \alpha+\log (1+(\beta / \alpha))=\log \alpha+O(\beta / \alpha)
$$

as $\beta / \alpha \rightarrow 0$, we have

$$
\begin{aligned}
x_{0, m+n}-x_{1, m+n} & =-\log \left[\left({ }^{m+n} Y^{1}\right)_{1,1} /\left({ }^{m+n} Y^{2}\right)_{1,1}\right] \\
& =-\log \alpha+O\left(\left(1-C^{-3}\right)^{n}\right)
\end{aligned}
$$

uniformaly in $m$ and $\omega$.

Since $\log \alpha$ is $\mathscr{M}_{0}^{n}$-measurable, we can easily obtain

$$
\begin{aligned}
& \left|\left(x_{0, t}-x_{1, t}\right)-E\left(x_{0, t}-x_{1, t} \mid \mathscr{M}_{0}^{n}\right)\right| \\
& \quad= \begin{cases}0, & (t \leqq n), \\
O\left(\left(1-C^{-3}\right)^{n}\right), & (t>n),\end{cases}
\end{aligned}
$$

uniformly in $t$ and $\omega$. This shows that the condition (4) of Theorem 1 holds. Therefore we can apply Theorem 1 to the random variables $x_{0, t}$ defined by $(3 \cdot 1)$. Consequently, we get the result of Theorem 2 for $i=j=1$.

To deal with the other values of $(i, j)$, note first that the inequality

$$
\left|\log \left({ }^{t} Y^{1}\right)_{i, j}-\log \left({ }^{t} Y^{1}\right)_{1,1}\right| \leqq 2 \log C
$$

follows from $(3 \cdot 4)$, as was pointed out in [1]. Therefore random variables

$$
\frac{1}{\sqrt{t}}\left\{\log \left({ }^{t} Y^{1}\right)_{1,1}-E\left(\log \left({ }^{t} Y^{1}\right)_{1,1}\right)\right\}
$$

and

$$
\frac{1}{\sqrt{t}}\left\{\log \left({ }^{t} Y^{1}\right)_{i, j}-E\left(\log \left({ }^{t} Y^{1}\right)_{i, j}\right)\right\}, \quad(1 \leqq i, j \leqq k)
$$

have an asymptotically same distribution. This completes the proof. 
By using Remarks 1 and 2 in $\S 1$ instead of Theorem 1, we can get the following Remarks 4 and 5 respectively. Their proofs can be copied from the previous one.

Remark 4. The conclusion of Theorem 2 remains valid if the conditions (2) and (3) of Theorem 2 are replaced by

(2') $\sum_{n=1}^{\infty}[\alpha(n)]^{\frac{\delta}{2+\delta}}<+\infty$ for some $\delta>0$,

(3') $E\left|\log \left(Z^{1}\right)_{1,1}\right|^{2+\delta}<+\infty$.

Remark 5. The conclusion of Theorem 2 remains valid again if the conditions (2) and (3) of Theorem 2 are replaced by

$\left(2^{\prime \prime}\right) \quad \sum_{n=1}^{\infty} \alpha(n)<+\infty$

$\left(3^{\prime \prime}\right) \log \left(Z^{1}\right)_{1,1}$ is an essentially bounded random variable.

Remark 6. It is easy to see that the assumption AII in [1], p. 464, is stronger than our condition (2) of Theorem 2, so that our results cover Furstenberg and Kesten's central limit theorem in [1].

\section{References}

[1] Furstenberg, H. and Kesten, H., Products of random matrices, Ann. Math. Statist., 31 (1960), 457-469.

[2] Ibragimov, I. A. and Linnik, Yu. V., Independent and stationary sequences of random variables, Wolters-Noordhoff, Gröningen, 1971.

[3] Kingman, J. F. C., The ergodic theory of subadditive stochastic process, J. Roy. Statist. Soc. Ser. B, 30 (1968), 499-510.

[4] Kingman, J. F. C., Subadditive ergodic theory, Ann. Probability, $\mathbb{1}(1973)$, 883909. 
